\title{
Índice de estado trófico e qualidade da água na produção de tilápias em tanques- rede em represa rural na região da Mata Atlântica na América do Sul
}

\author{
Leonardo, A.F. ${ }^{1}$; Baccarin, A.E. ${ }^{2}$; Trombeta, T.D. ${ }^{3}$; Machado, L.P. ${ }^{4}$; Teramoto, E.T. ${ }^{4}$ e Bueno, G.W. ${ }^{4}$
}

\begin{abstract}
'Agência Paulista de Tecnologia dos Agronegócios. Registro. SP. Brasil.
${ }^{2}$ Secretaria de Meio Ambiente de São Paulo. Registro. SP. Brasil.

${ }^{3}$ Universidade Federal do Oeste do Pará - UFOPA. Monte Alegre. PA. Brasil.

${ }^{4}$ Universidade Estadual Paulista - Unesp. Registro. SP. Brasil.
\end{abstract}

\section{PALAVRAS CHAVE ADICIONAIS}

Aquicultura sustentável.

Sistema intensivo.

Boas praticas de manejo.

Qualidade de água.

\section{RESUMO}

A produção de tilápias (Oreochromis niloticus) em tanques-rede exige o constante monitoramento das propriedades físico-químicas da água, pois é uma atividade considerada potencialmente polvidora do ambiente aquático e o monitoramento é fundamental para evitar processos de eutrofização do corpo hídrico. O presente estudo avaliou a influência da produção intensiva de tilápia do Nilo em tanques-rede sobre o grau de trofia e qualidade da água em uma represa localizada no Vale do Ribeira, região de Mata Atlântica no sudeste do Brasil. Na classificação do grau de trofia foram considerados cinco classes, que foram estabelecidas a partir do Índice do Estado Trófico (IET). O experimento foi conduzido em três períodos: antes (90 dias), durante (180 dias) e após (90 dias) à produção intensiva de peixes na represa. Instalaram-se 30 tanques-rede de $4 \mathrm{~m}^{3} \mathrm{com}$ densidade de estocagem de 150 peixes por $\mathrm{m}^{3}$. A partir dos resultados experimentais, verificouse que o IET foi crescente no período de produção passando de ultraoligotrófico à mesotrófico, alcançou valor máximo de 53,2 $\pm 0,4$ e após a produção manteve-se oligotrófico. Os parâmetros de qualidade de água não apresentaram diferenças significativas entre os períodos analisados (P>0.05). A aplicação com base no IET representa uma ferramenta prática para o monitoramento ambiental e análise de riscos da produção de peixes no ambiente aquático.

\section{IET for the environmental monitoring of the intensive production of tilapia in cage} in the Atlantic Forest region of South America

\section{ADDITIONAL KEYWORDS}

Sustainable aquaculture.

Intensive system.

Good management practices.

Water quality.

\section{INFORMATION}

Cronología del artículo.

Recibido/Received: 01.12 .2016

Aceptado/Accepted: 15.03.2018

On-line: 15.04 .2018

Correspondencia a los autores/Contact e-mail:

guilherme.wolff@unesp.br

\section{SUMMARY}

The production of tilapia (Oreochromis niloticus) in cages requires the constant monitoring of the physical and chemical water properties, since it is an activity considered potentially polluting for the aquatic environment and its monitoring is fundamental to avoid the water body eutrophication processes. The present study was conducted in a reservoir located in a fishfarm at Pariquera - Açu County (São Paulo state, Brazil) and evaluated the influence of the intensive Nile tilapia production in cages on the trophic state and in the water quality. For the trophic state determination, five classes were considered and stablished by the Trophic State Index (TSI). The trial was developed and divided in three phases: before (90 days), during (180 days) and after (90 days) the fish intensive production in the reservoir. Thirty net cages with volume of $4 \mathrm{~m}^{3}$ and stocking density of 150 fish per $\mathrm{m}^{3}$ were installed. According to the results, it was observed that the trophic state index (TSI) was increasing according to the production period, changing from an ultra-oligotrophic to a mesotrophic state, reaching a maximum value of $53.2 \pm 0.4$, and, after production period, remained oligotrophic. The water quality parameters did not present significant differences between the periods analysed $(P>0.05)$. The implementation of the TSI represents a practical tool for environmental monitoring and risk analysis of fish production in the aquatic environment.

\section{INTRODUÇÃO}

A criação de tilápias em tanques-rede ou gaiolas flutuantes é uma tecnologia que oferece alta produtividade (1.500 a $2.000 \mathrm{t}$ de peixes / ha) e rápido retorno de investimento (payback descontado em média de 2 anos), fato que tem atraído novos empresários e investidores, principalmente em países em desenvolvimento, onde se faz necessário o aumento da produção de alimento para a garantia da segurança alimentar e geração de recursos financeiros (Béné et al., 2016; Godoy et al., 2016).

Neste contexto, a produção de tilápias em tanques-rede favorece o melhor aproveitamento dos recursos hídricos disponíveis, como reservatórios, rios e lagos, permitindo a troca completa de água dentro dos tanques-rede várias vezes ao dia, o que proporciona 
oxigenação aos peixes e dispersão dos metabólitos produzidos, principalmente resíduos fosfatados e nitrogenados (Bureau e Hua, 2010; Gondwe et al., 2011; Canale et al., 2016).

No entanto, é uma atividade considerada potencialmente poluidora e pode acarretar acúmulo de matéria orgânica e aumento dos nutrientes no ambiente aquáti$\mathrm{co}$, principalmente de nitrogênio e fósforo, produto da excreção dos peixes e sobras de ração (Macedo et al., 2010; Bueno et al., 2017; Rabassó e Hernández, 2015; Cai et al., 2016). Soma-se a isso, o lançamento de esgoto doméstico e industrial, o uso de fertilizantes e agrotóxicos na agricultura, que quando lixiviado ocasionam a contaminação da água a qual pode ser agravada com o desmatamento das matas ciliares devido à intensificação do assoreamento e erosão no corpo hídrico.

Portanto, o excesso de nutrientes no corpo hídrico causa a eutrofização, podendo aumentar a concentração de fitoplâncton na água, diminuir os níveis de oxigênio dissolvido e desencadear o aparecimento de substancias tóxicas no meio aquático (Tovar, 2000; Godoy et al., 2016). Nesse contexto, é fundamental o desenvolvimento da aquicultura sustentável e ecologicamente correta, estabelecendo efetivo monitoramento das condições do ambiente aquático e o uso de ferramentas específicas para a criação de tilápias em tanques-rede, as quais irão auxiliar na regulamentação, monitoramento ambiental e dimuição de riscos aos empreendimentos aquícolas e ao ecossitema aquático (Barton e Fløysand, 2010; Montanhini Neto e Ostrensky, 2015).

Dessa forma, além do monitoramento físico-químico da qualidade da água, o uso de indicadores é considerado uma importante ferramenta a ser utilizada no monitoramento e controle da eutrofização do corpo hídrico, dentre estas se destaca o Índice de Estado Trófico (IET), desenvolvido com a finalidade de classificar as águas de lagos e reservatórios (Lamparelli, 2004; Bueno et al., 2008; ANA, 2014). Este índice divide os sistemas tradicionais em três classes: oligotrófico, mesotrófico e eutrófico, sendo que determinados sistemas incluem, também classes como o ultraoligotrófico, hipereutrófico, entre outras que são baseados na concentração de fósforo e clorofila- $a$ no ambiente aquático (Carlson, 1977).

Diante do exposto, o objetivo do presente estudo foi avaliar a influência da produção intensiva de tilápia do Nilo em tanques-rede sobre o grau de trofia e qualidade da água em uma represa localizada no Vale do Ribeira, Brasil. O IET foi utilizado na classificação do grau de trofia, a fim de verificar sua aplicabilidade como ferramenta no levantamento dos impactos ambientais da piscicultura intensiva em tanques-rede. $\mathrm{O}$ Vale do Ribeira é considerada uma importante região devido à sua biodiversidade presente em ecossistemas aquáticos e terrestres, pois abriga aproximadamente $53 \%$ da Mata Atlântica remanescente no Brasil, sendo a principal área com este bioma na América do Sul.

Atualmente, um dos grandes desafios para a região é a expansão e o desenvolvimento sustentável da agricultura e da aquicultura, que devem ser realizadas aproveitando de maneira racional os recursos naturais locais e trazendo desenvolvimento social e econômico.

\section{MATERIAL E MÉTODOS}

O trabalho foi realizado, no setor de Piscicultura do Pólo Regional do Vale do Ribeira, da Agência Paulista de Tecnologia dos Agronegócios (APTA Regional), localizado no município de Pariquera-Açú (latitude $24^{\circ} 43^{\prime} 14^{\prime \prime} \mathrm{S}$; longitude $47^{\circ} 52^{\prime} 43^{\prime \prime} \mathrm{O}$; altitude $39 \mathrm{~m}$ ). O município apresenta clima equatorial úmido (Af pela Classificação de Köppen-Geiger), com temperatura média anual de $22.3^{\circ} \mathrm{C}$ e precipitação acumulada anual de $1900.8 \mathrm{~mm}$. A represa utilizada no estudo apresenta uma área de $20.700 \mathrm{~m}^{2}$ de superfície de água, com profundidade média de $5 \mathrm{~m}$ e vazão média no período da seca (outono e inverno) de $0,010 \mathrm{~m}^{3} \mathrm{~s}^{-1}$, e no período das chuvas (primavera e verão) com vazão média de 0.028 $\mathrm{m}^{3} \mathrm{~s}^{-1}$. Instalaram-se três linhas com dez tanques-redes de $4 \mathrm{~m}^{3}$, totalizando 30 tanques-redes com espaçamento entre tanques de dois metros. Utilizou-se densidade de estocagem de 150 peixes por $\mathrm{m}^{3}$ e tilápias do Nilo com peso médio de $5 \pm 2.6 \mathrm{~g}$. Ao final do período experimental foram despescados $7.169,95 \mathrm{~kg}$ de peixe.

A ração comercial extrusada utilizada continha 32\% de proteína bruta, $15 \mathrm{MJ} \mathrm{kg}^{-1}$ de energia bruta e teor de $0.6 \%$ de fósforo total. Os peixes foram alimentados duas vezes ao dia na proporção média de $2.5 \%$ do peso vivo onde a quantidade fornecida foi corrigida mensalmente após as biometrias de 5\% dos indivíduos. Portanto, o total de ração utilizado durante o experimento foi de $10.249,70 \mathrm{~kg}$, com uma conversão alimentar média de 1.40 .

O experimento foi conduzido em três períodos: antes, durante e depois da produção de peixes, sendo: junho a agosto (90 dias), outubro a março (180 dias) e abril a junho (90 dias), respectivamente. Durante este período, monitoraram-se semanalmente os níveis de oxigênio dissolvido (OD mg L $\mathrm{L}^{-1}$ ) e temperatura da água $\left({ }^{\circ} \mathrm{C}\right)$ utilizando-se o aparelho portátil YSI 550A, além do potencial hidrogeniônico com o medidor de $\mathrm{pH}$ digital HANNA-21 e condutividade elétrica $\left(\mu S . \mathrm{cm}^{-1}\right)$, utilizando-se o condutivímetro de bancada ADAMO C-150. A transparência da água $(\mathrm{cm})$ foi medida com o Disco de Secchi.

Paralelamente, utilizando uma garrafa de Van Dorn, coletaram-se, mensalmente, amostras de água no abastecimento da represa $(\mathrm{AB})$ a $40 \mathrm{~cm}$ da superfície $(n=3)$, na área onde estavam instalados os tanquesrede (TR) a $100 \mathrm{~cm}$ da superfície $(n=9)$ e no efluente de lançamento (EF) da represa a $40 \mathrm{~cm}$ da superfície $(n=3)$, ambas em triplicata. Em seguida, as amostras foram acondicionadas em garrafas plásticas, armazenadas na ausência de luz e refrigeradas a $4^{\circ} \mathrm{C}$ para posteriores análises laboratoriais.

A determinação da alcalinidade total $\left(\mathrm{CaCO}_{3} \mathrm{mg}\right.$ $\mathrm{L}^{-1}$ ) foi realizada por meio de titulação com acido forte (Golterman et al., 1978) e a concentração de nutrientes dissolvidos foi realizada por meio da filtragem das amostras de água em membrana do tipo GF/C Whatman $(0.45 \mu \mathrm{m}$ de porosidade, $47 \mathrm{~mm}$ de diâmetro), sendo posteriormente congeladas para análise das 
concentrações de nitrogênio amoniacal total $\left(\mathrm{mg} \mathrm{L}^{-1}\right)$ de acordo com o proposto por Koroleff (1976). A análise do material em suspensão $\left(\mathrm{mg} \mathrm{L}^{-1}\right)$ seguiu o método de Mudroch e Macknight (1991) e para a clorofila- $a$ ( $\left.\mu \mathrm{g} \mathrm{L}^{-1}\right)$ o método de Nusch (1980) e Marker et al. (1980).

Nos mesmos pontos de coleta da água, obtiveramse amostras de sedimento por meio de uma draga de Ekman. Após a coleta, as amostras foram preparadas e armazenadas, seguindo a metodologia do Instituto Agronômico de Campinas (IAC) do Centro de Solos e Recursos Ambientais.

Amostras de água não filtradas também foram congeladas para posterior análise da bioacumulação de P-total na água e no sedimento, nos três períodos estudados, onde seguiu-se o proposto por Golterman et al. (1978) por meio do método de leitura em ultravioleta visível (UV-vis). A partir dos resultados obtidos para as concentrações de P-total e Clorofila- $a$ determinou-se o índice de eutrofização do ambiente aquático.

O Índice do Estado Trófico foi composto pelo Índice do Estado Trófico para o Fósforo - IET (PT) e o Índice do Estado Trófico para a Clorofila- $a$ - IET (CL), modificados por Lamparelli (2004), sendo estabelecidos para ambientes lóticos, segundo as equações:

$$
\begin{aligned}
& \text { IET (Cl): } \left.10^{*}\left(6-\left(0.92-0.34^{*}(\operatorname{In~Cl})\right) / \text { In } 2\right)\right) \text {; } \\
& \text { IET (PT): } 10^{*}(6-(1.77-042 *(\operatorname{In~PT)~/~In~} 2)) \text {; } \\
& \text { onde: }
\end{aligned}
$$

$\mathrm{Cl}$ : concentração de Clorofila- $a$ medida à superfície da água, em $\mu g . L^{-1}$;

PT: concentração de Fósforo Total medida à superfície da água, em $\mu \mathrm{g} . \mathrm{L}^{-1}$;

ln: logaritmo natural.
O resultado apresentado nas tabelas do IET foi à média aritmética simples dos índices relativos ao Fósforo Total e a Clorofila $a$, segundo a equação:

$$
\operatorname{IET}=[\operatorname{IET}(\text { PT })+\operatorname{IET}(\text { CL) }] / 2
$$

Foi utilizada a classificação numérica descrita por Lamparelli (2004), a qual classifica os ambientes como: ultraoligotrófico (IET $\leq 47)$, oligotrófico $(47<$ IET $\leq 52)$, mesotrófico $(52<$ IET $\leq 59)$, eutrófico $(59<$ IET $\leq 63)$, supereutrófico $(63<$ IET $\leq 67)$ e hipereutrofico $(>67)$. Para se verificar a ocorrência de estratificação foi realizada análise de variância de médias repetidas utilizando-se o tempo (meses) e como repetição os pontos de coleta, em caso de diferença significativa as médias foram comparadas pelo teste de Tukey $(\mathrm{p}<0.05)$.

\section{RESULTADOS E DISCUSSÃO}

Os resultados para as variáveis físicas e químicas da água como temperatura (TP), oxigênio dissolvido (OD), potencial hidrogeniônico $(\mathrm{pH})$, condutividade elétrica (CE), alcalinidade total (AT) e nitrogênio amoniacal total (NT) são apresentadas na Tabela I. Verificase que os valores médios atenderam aos requisitos para a criação de tilápias de acordo com o recomendado por New (1990) e Leonardo et al. (2011) e não apresentaram diferença significativa $(\mathrm{P}>0,05)$ entre os pontos de coleta no abastecimento da represa (AB), local de instalação dos tanques-rede (TR) e no efluente de lançamento (EF) e entre os três períodos do experimento (antes, durante e após a produção de tilápia).

Os valores de qualidade da água são bastante similares entre os pontos de coleta AB, TR e EF (Tabela I). No entanto, entre os períodos de coleta, observou-se

\begin{tabular}{|c|c|c|c|c|c|c|c|}
\hline Período & $\begin{array}{l}\text { Ponto de } \\
\text { coleta }\end{array}$ & $\begin{array}{l}\text { TP } \\
\left({ }^{\circ} \mathrm{C}\right)\end{array}$ & $\begin{array}{c}\mathrm{OD} \\
\left(\mathrm{mg} \mathrm{L}^{-1}\right)\end{array}$ & $\mathrm{pH}$ & $\begin{array}{c}\text { CE } \\
\left(\mu \mathrm{cm}^{-1}\right)\end{array}$ & $\begin{array}{c}\mathrm{AT} \\
\left(\mathrm{CaCO}_{3} \mathrm{mg} \mathrm{L}^{-1}\right)\end{array}$ & $\begin{array}{c}\mathrm{NT} \\
\left(\mathrm{mg} \mathrm{L}^{-1}\right)\end{array}$ \\
\hline \multirow{3}{*}{$\begin{array}{l}\text { Antes } \\
\text { da Produção }\end{array}$} & $A B$ & $20.7 \pm 0.2^{\mathrm{ac}}$ & $7.4 \pm 0.3^{\mathrm{aA}}$ & $6.5 \pm 0.2^{\mathrm{aA}}$ & $42.1 \pm 1.0^{\mathrm{aA}}$ & $17.1 \pm 0.5^{\mathrm{aA}}$ & $0.014 \pm 0.002^{\mathrm{aA}}$ \\
\hline & TR & $20.7 \pm 0.3^{\mathrm{ac}}$ & $7.4 \pm 0.4^{\mathrm{aA}}$ & $6.4 \pm 0.1^{1 \mathrm{~A}}$ & $43.0 \pm 0.8^{\mathrm{aA}}$ & $17.1 \pm 0.1^{\mathrm{aA}}$ & $0.016 \pm 0.003^{\mathrm{aA}}$ \\
\hline & EF & $20.8 \pm 0.2^{\mathrm{ac}}$ & $7.4 \pm 0.4^{\mathrm{aA}}$ & $6.2 \pm 0.3^{\mathrm{aA}}$ & $42.5 \pm 0.5^{\mathrm{aA}}$ & $17.0 \pm 0.2^{\mathrm{aA}}$ & $0.013 \pm 0.003^{\mathrm{aA}}$ \\
\hline \multirow{3}{*}{ Produção } & $A B$ & $26.3 \pm 0.2^{\mathrm{aA}}$ & $7.8 \pm 0.4^{\mathrm{aA}}$ & $6.2 \pm 0.2^{\mathrm{aA}}$ & $45.1 \pm 0.3^{\mathrm{aA}}$ & $17.2 \pm 0.2^{\mathrm{aA}}$ & $0.015 \pm 0.003^{\mathrm{aA}}$ \\
\hline & TR & $26.1 \pm 0.1^{\mathrm{aA}}$ & $7.6 \pm 0.6^{\mathrm{aA}}$ & $6.1 \pm 0.3^{\mathrm{aA}}$ & $45.3 \pm 0.1^{\mathrm{aA}}$ & $17.4 \pm 0.3^{\mathrm{aA}}$ & $0.020 \pm 0.004^{\mathrm{bB}}$ \\
\hline & EF & $26.0 \pm 0.2^{\mathrm{aA}}$ & $7.0 \pm 0.8^{\mathrm{aA}}$ & $6.1 \pm 0.3^{\mathrm{aA}}$ & $45.0 \pm 0.4^{\mathrm{aA}}$ & $17.0 \pm 0.3^{\mathrm{aA}}$ & $0.015 \pm 0.003^{\mathrm{aA}}$ \\
\hline \multirow{3}{*}{$\begin{array}{l}\text { Pós- } \\
\text { Produção }\end{array}$} & $A B$ & $22.3 \pm 0.2^{\mathrm{aB}}$ & $12.2 \pm 0.5^{\mathrm{aB}}$ & $6.9 \pm 0.1^{\mathrm{aA}}$ & $42.7 \pm 0.3^{\mathrm{aA}}$ & $17.0 \pm 0.8^{\mathrm{aA}}$ & $0.013 \pm 0.003^{\mathrm{aA}}$ \\
\hline & TR & $22.1 \pm 0.1^{\mathrm{aB}}$ & $12.2 \pm 0.6^{\mathrm{aB}}$ & $7.0 \pm 0.2^{\mathrm{aA}}$ & $43.0 \pm 0.1^{\mathrm{aA}}$ & $17.1 \pm 0.7^{\mathrm{aA}}$ & $0.013 \pm 0.005^{\mathrm{aA}}$ \\
\hline & EF & $22.0 \pm 0.2^{\mathrm{aB}}$ & $12.7 \pm 0.3^{\mathrm{aB}}$ & $6.9 \pm 0.1^{\mathrm{aA}}$ & $43.1 \pm 0.1^{\mathrm{aA}}$ & $17.2 \pm 0.6^{\mathrm{aA}}$ & $0.013 \pm 0.002^{\mathrm{aA}}$ \\
\hline \multicolumn{8}{|c|}{$\begin{array}{l}\text { Médias seguidas de mesma letra nas colunas não diferem estatisticamente pelo teste de Tukey a } 5 \% \text {, não houve diferença estatística } \\
\text { entre linhas. } \\
\text { Temperatura da água (TP), oxigênio dissolvido (OD), pH, condutividade elétrica (CE), alcalinidade total (AT), nitrogênio amoniacal total } \\
\text { (NT), abastecimento (AB), tanque-rede (TR) e efluente (EF }\end{array}$} \\
\hline
\end{tabular}
um aumento de $5^{\circ} \mathrm{C}$ em relação as médias de temperatura da água no período (outubro a março) e nas estações climáticas da primavera e verão um decréscimo 
de $4^{\circ} \mathrm{C}$ influenciada pelas estações de outono e inverno, no período de pós-produção. Essa variação é similar ao encontrado por Romagosa et al. (2003), Leonardo et al. (2006) e Dias et al. (2012) em estudos com peixes tropicais realizados na mesma região. Fato que influência no desempenho zootécnico dos animais pecilotérmicos e consequentemente no consumo de ração, excreção e qualidade da água no ambiente de produção.

Os níveis mais elevados de oxigênio dissolvido, acima de $12 \mathrm{mg} \mathrm{L}^{-1}$, foram observados no período de pós-produção, que ocorreu de abril a junho, nas estações climáticas outono e inverno. Segundo Wetzel (2001) e Hennemann e Petrúcio (2011) esse fenômeno ocorre devido à diminuição de temperatura da água, provocando maior solubilidade do oxigênio, fatores que corroboram com o observado na Tabela I.

Entretanto, o pH, CE, AT e NT não apresentaram oscilações durante o período experimental ( $\mathrm{P}>0.05)$, resultado semelhante aos estudos de Bueno, et al. (2008); Américo, et al. (2013) e Garcia, et al. (2013) que também não encontraram diferenças significativas nos parâmetros de qualidade de água em locais onde se instalaram tanques-rede para a produção intensiva de peixes em reservatórios, valores que demonstram a necessidade do uso de ferramentas e indicadores que permitam o monitoramento e acompanhamento constante da evolução da qualidade da água destes ambientes aquáticos, e não somente em determinadas épocas definidas por coletas e análises pontuais no tempo.

Outro fator de estrema importância para a sustentabilidade da produção e do ecossistema aquático tratase da produção de resíduos metabólicos de nitrogênio $(\mathrm{N})$ e fósforo $(\mathrm{P})$ lançados pelos peixes os quais são determinados por diversos fatores endógenos e exógenos, como genética, idade, biomassa, ambiente de criação e qualidade e teor de nutrientes da dieta (Gondwe et al., 2011; Canale et al., 2016). Segundo Cai et al. (2016) esses compostos são os principais produtos de excreção dos peixes, podendo prejudicar a qualidade da água da criação. Ao avaliarmos os valores de nitrogênio amoniacal total (Tabela I), verificaram-se o acréscimo médio de $20 \%$ na concentração de NT durante e antes a produção dos peixes, portanto, não se pode afirmar que o input de rações e excretas oriundo da produção de peixes influenciou na concentração de nitrogênio no ambiente aquático durante o estudo. Além disso, ao compararmos aos limites estabelecidos pela Resolução do Conselho Nacional de Meio Ambiente - Conama $\mathrm{n}^{\mathrm{o}} 357$, de $17 / 03 / 2005$, verificam-se que os valores encontrados estão abaixo dos limites de lançamento de efluentes em água doce de Classe $01\left(3,7 \mathrm{mg} \mathrm{L}^{-1} \mathrm{~N}\right.$, para $\mathrm{pH} \leq 7.5)$.

Em relação à transparência da água, houve diferença estatística $(\mathrm{P}<0.023)$ no ponto de coleta dos tanques-rede, conforme mostrado na Tabela II a qual pode ser influenciada pelo aporte de matéria orgânica no ambiente que, consequentemente, libera nutrientes que contribuíram com o desenvolvimento da comunidade fitoplanctônica e aumento de material particulado suspenso. Apesar dessas diferenças (63 a $103 \mathrm{~cm}$ ), a transparência da água durante a fase experimental manteve-se próximo aos padrões normais para criação de tilápias em tanques-rede que variam de 50 a $200 \mathrm{~cm}$ (Popma e Lovshin, 1996; Kubtiza, 2000; Bueno et al., 2008; Garcia et al., 2013). Verificou-se também que o ponto do abastecimento obteve-se média de $100 \mathrm{~cm}$, durante todo o experimento, demonstrando que a cargas de nutrientes oriundas da água de abastecimento da represa não influenciaram o ambiente em estudo ao longo do tempo.

Ao avaliarmos o grau de trofia do ambiente (IET) demonstrado na Tabela III, verifica-se que a represa no período antes da produção, pontos de coleta $\mathrm{AB}$ e EF o ambiente classificou-se como ultraoligotrófico. No entanto, no período de produção o grau de trofia nos pontos de coleta $\mathrm{AB}$ e TR foram alterados para mesotrófico. Contudo, no período de pós-produção, o grau de trofia apresentou decréscimo para oligotrófico. Estes resultados reforçam o recomendado pela Agência Nacional de Águas - ANA (2014) que enfatizam o uso do IET para avaliação continua da qualidade da água e do potencial de risco de impacto ambiental em reservatórios e represas relacionados à exploração dos recursos hídricos, inclusive àqueles destinados a produção animal os quais necessitam de outorga de água e licenciamento ambiental para instalação e operação do empreendimento.

Neste contexto, a mudança de classificação do IET no período de produção observada neste estudo, pode estar atribuída ao aporte de matéria orgânica (MO), oriunda da excreção dos peixes ou mesmo de ração não digerida. Além destes fatores, a precipitação observada neste período de $1080 \mathrm{~mm}$ por ano, típico da região de Mata Atlântica, pode contribuir com o lixiviamento de $\mathrm{MO}$, nutrientes e consequentemente aumento na concentração de clorofila- $a$ no ambiente aquático em estudo. Zhang et al. (2008) também utilizando índice de estado trófico (baseado na concentração de nitrogê-

Tabela II. Valores médios (média \pm desvio padrão) da transparência da água $(\mathrm{cm})$ durante o experimento (Mean values (mean \pm standard deviation) of water transparency $(\mathrm{cm})$ in the experimente).

\begin{tabular}{|c|c|c|c|}
\hline Ponto de coleta & Antes da Produção & Produção & Pós-Produção \\
\hline Abastecimento & $100 \pm 0.01^{a}$ & $103 \pm 35.0^{\mathrm{a}}$ & $100 \pm 0.10^{a}$ \\
\hline Tanques-rede & $73.0 \pm 23.0^{\mathrm{b}}$ & $63.3 \pm 5.4^{b}$ & $73.3 \pm 23^{b}$ \\
\hline Efluente & $92.3 \pm 0.01^{\mathrm{a}}$ & $96.7 \pm 0.07^{a}$ & $98.0 \pm 0.05^{\mathrm{a}}$ \\
\hline
\end{tabular}

Médias seguidas de mesma letra nas colunas não diferem estatisticamente pelo teste de Tukey a 5\%, não houve diferença estatística entre linhas. 
Tabela III.Valores médios (média \pm desvio padrão) do Índice do Estado Trófico (IET) durante o período experimental (Mean values (mean \pm standard deviation) of the Trophic State Index (EIT) of water in the experimental).

\begin{tabular}{|c|c|c|c|}
\hline Ponto de coleta & Antes da Produção & Produção & Pós-Produção \\
\hline Abastecimento & $44.9 \pm 0.4^{c}$ & $52.3 \pm 0.8^{a}$ & $49.9 \pm 0.1^{b}$ \\
\hline Tanques-rede & $47.3 \pm 0.6^{c}$ & $53.2 \pm 0.4^{a}$ & $48.1 \pm 0.3^{b}$ \\
\hline Efluente & $46.2 \pm 0.7^{c}$ & $50.0 \pm 0.5^{\mathrm{a}}$ & $48.2 \pm 0.2^{b}$ \\
\hline
\end{tabular}

Médias seguidas de mesma letra nas colunas não diferem estatisticamente pelo teste de Tukey a 5\%, houve diferença estatística entre linhas.

nio, fósforo e clorofila- $a$ na água) para avaliar a eutrofização em 35 reservatórios na região de Chongqing, no Sudoeste da China enfatizaram a eficiência do uso desta ferramenta a qual permitiu a conclusão que as principais fontes de poluição são os resíduos industriais e domésticos, sendo intensificado pelo desenvolvimento da aquicultura onde o nível de eutrofização aumentou em dois anos gradualmente de ultraoligotrófico para oligotrófico em 22 reservatórios.

Contudo, o uso do IET e de indicadores de qualidade da água tem sido utilizado com frequência para o monitoramento ambiental de reservatórios, lagos e represas para fins de licenciamento e controle ambiental (ANA, 2014). No entanto, a aplicação destas ferramentas em áreas de produção intensiva de peixes é incipiente e deve ser aplicada com maior frequência pela indústria aquícola, pois trata-se de uma metodologia prática e pouco onerosa que permite resultados de fácil interpretação e uso pelos produtores, agentes fiscalizadores e investidores no setor que visam uma atividade sustentável e com menor risco.

Em relação ao fósforo total dissolvido na água, os valores observados no estudo se mantiveram abaixo de $0.020 \mathrm{mg} \mathrm{L}^{-1}$, limite estabelecido pela Resolução Conama $\mathrm{n}^{\circ} 357$ de 17/03/05 para água doce. Entretanto, de acordo com Boyd e Tucker (1998), para um bom desenvolvimento dos organismos aquáticos, as concentrações de fósforo total nos sistemas de piscicultura devem ser menores que $0.5 \mathrm{mg} \mathrm{L}^{-1}$. Esteves (1998) ressalta que outra forma de avaliar o fósforo é por meio da coleta de sedimento, devido a sua integração com todo processo que ocorre no ambiente aquático, ocorrendo os processos biológicos, físicos e químicos.

Segundo Nunes et al., (2013) os fatores bióticos e abióticos estão envolvidos na dinâmica do fósforo no ecossistema aquático. Além disso, a estratégia alimentar, em criações comerciais de peixes, possui importante impacto sobre a qualidade da água e, consequentemente, sobre o crescimento, saúde e sobrevivência dos animais, assim como a eficiência de utilização dos nutrientes da dieta.

Os resultados obtidos demonstram a dinâmica hídrica da represa, que na época das secas possuem uma vazão média de $0.010 \mathrm{~m}^{3} \mathrm{~s}^{-1}$ e na época de chuvas possuem uma vazão média de $0.028 \mathrm{~m}^{3} \mathrm{~s}^{-1}$, ocasionado, possivelmente, o carreamento de fósforo no ponto efluente, valores que corroboram com o observado por Malheiros (2012) e Nunes et.al (2013). Em relação ao material em suspensão, durante toda fase experimental, mesmo havendo uma diferença estatística $(P>0.043)$ entre alguns pontos de coletas, os valores observados durante o estudo estiveram abaixo de $2.0 \mathrm{~g} \mathrm{~L}^{-1}$, os quais são recomendado para criação de peixes de acordo com Leonardo et al. (2011).

Contudo, o uso do indicador IET como ferramenta de monitoramento ambiental aplicado nesse estudo demonstrou-se de forma prática e precisa uma abordagem a ser utilizada pelo setor aquícola em fazendas de produção de peixes em reservatórios ou represas visando à mitigação de riscos de impactos ambientais decorrentes da produção intensiva em ambientes aquáticos, de atividades agrícolas e industriais que impactam o corpo hídrico e podem influenciar na sustentabilidade da aquicultura e do ambiente aquático.

\section{CONCLUSÃO}

O ambiente aquático estudado classifica-se como ultraoligotrófico, antes da criação de peixes, em mesotrófico durante a criação e oligotrófico após a criação de peixes. A aplicação do IET como uma ferramenta para o monitoramento ambiental da produção intensiva de peixes é uma ferramenta prática que pode ser utilizada para gestão de reservatórios e represas utilizados para a aquicultura.

Tabela IV. Valores médios (média \pm desvio padrão) do material em suspensão na água (Mean values (mean \pm standard deviation) of suspended material in water of cages area).

\begin{tabular}{lccc}
\hline Ponto de Coleta & Antes da Produção & Produção & Pós-Produção \\
\hline Abastecimento & $0.012 \pm 0.002^{\mathrm{B}}$ & $0.016 \pm 0.002^{\mathrm{bA}}$ & $0.007 \pm 0.001^{\mathrm{cC}}$ \\
Tanques-rede & $0.013 \pm 0.001^{\mathrm{C}}$ & $0.028 \pm 0.007^{\mathrm{aA}}$ & $0.021 \pm 0.002^{\mathrm{aB}}$ \\
Efluente & $0.014 \pm 0.001^{\mathrm{B}}$ & $0.021 \pm 0.004^{\mathrm{CA}}$ & $0.014 \pm 0.003^{\mathrm{bB}}$ \\
\hline
\end{tabular}

Médias seguidas de mesma letra minúscula nas colunas não diferem estatisticamente pelo teste de Tukey a 5\%. 


\section{AGRADECIMENTOS}

A Fundação de Amparo à Pesquisa do Estado de São Paulo (FAPESP), Processo n.2016/10563-0 pelo auxílio na pesquisa. A FUNDEPAG, FEHIDRO e aos técnicos do setor de Piscicultura do Polo Regional do Vale do Ribeira Sr. Edilberto Rufino de Almeida, Benedito de Aguiar Martins e André Luis Aguiar Martins pelo auxílio na execução do projeto de pesquisa.

\section{BIBLIOGRAFIA}

Agência Nacional de Águas - ANA 2014. Indicadores de qualidade: índice do estado trófico (IET). Disponível em http:// portalpnqa.gov. $\mathrm{br} /$ indicadores-estado-trofico.aspx. Acesso em: 15 nov 2016.

Américo, JHP, Previato, Vand de Carvalho, SL 2013. Qualidade da água de uma piscicultura em tanques-rede no rio São José dos Dourados, Ilha solteira - São Paulo. Periódico Eletrônico Fórum Ambiental da Alta Paulista, 9(2).

Barton, JR and Fløysand, A 2010. The political ecology of Chilean salmon aquaculture, 1982-2010: A trajectory from economic development to global sustainability. Global Environmental Change, 20(4), 739-752. Béné, $C$, Arthur, $R$, Norbury, $H$, Allison, EH, Beveridge, $M$, Bush, $S$, Campling, L, Leschen, W, Little, D, Squires, D, Thilsted, SH, Troell, M, Williams, M 2016. Contribution of Fisheries and Aquaculture to Food Security and Poverty Reduction: Assessing the Current Evidence. World Development, v. 79, p.177-196.

Boyd, CE 1990. Water quality in ponds for aquaculture. Auburn: Auburn University Experimental Station. $482 \mathrm{p}$.

Boyd, CE, Tucker, CS 1998. Pond aquaculture water quality management. Boston: Kluwer Academic, 700 p.

Brasil 2005 Resolução n 357, de 17 de marco de 2005. Ministério do Desenvolvimento Urbano e Meio Ambiente. Conselho Nacional do Meio Ambiente. Publicação DOU.: 18/03/2005. Disponível em: <http.//www.mma.gov.br/port /conama> Acesso em: 05 out 2016.

Bueno, GW, Marengoni, NG, Gonçalves, ACJ, Boscolo, RW, Teixeira, RA 2008. Estado trófico e bioacumulação do fósforo total no cultivo de peixes em tanques-rede na área aquícola do reservatório de ltaipu Acta Scientiarum. Biological Sciences, Maringá-PR vol. 30, núm. 3, pp 237-243.

Bueno, GW, Bureau, DP, Skipper-Horton, JO, Roubach, R, De Matos, FT.; Bernal, FEM 2017. Mathematical modeling for the management of the carrying capacity of aquaculture enterprises in lakes and reservoirs. Pesquisa agropecuaria brasileira, v. 52, p.695- 705.

Bureau, DP, Hua, K 2010. Towards effective nutritional management of waste outputs in aquaculture, with particular reference to salmonid aquaculture operations. Aquaculture Research, v.41, p.777-792.

Cai, H, Ross, L.G, Telfer, T.C, Changwen, W, Zhu, A 2016. Environmental Science and Pollution Research, v.23, n.8, p.7529-7542.

Canale, RP, Whelan, G, SwitzeR, A, Eisch, E 2016. A bioenergetic approach to manage production and control phosphorus discharges from a salmonid hatchery. Aquaculture, v.451, n 20, p.137-146.

Carlson, R.E 1977. A trophic state index for lakes. Limnologic. Oceanograf, Baltimore, v 22, n 2, p. 361-369.

Dias, DC, Leonardo, AF, Tachibana, L, Correa, CF , Bordon, IA, Romagosa, E, Ranzani-Paiva, MJT 2012. Effect of incorporating into the diet of matrinxa, Brycon amizonicus, breeders. Journal of Applied Ichthyology, v 28, p. 40-45.

Esteves, FA1998. Fundamentos de limnologia 2. ed. Rio de Janeiro: Interciência

Garcia, F, Romera, DM, Gozi, KS, Onaka, EM, Fonseca, FS, Schalch, SHC, Candeira, PG, Guerra, LOM, Carmo, FJ, Carneiro,DJ, Martins, MIEG, Portella, MC 2013. Stocking density of Nile tilapia in cages placed in a hydroelectric reservoir. Aquaculture, v. 410, p. 51-56

Golterman, HL, Clymo, RS, Ohnstad, MAM 1978. Methods for physical and chemical analysis of freshwater. London: IBP, Blackwell Science. Public 213p.
Godoy, AC, Fries, E, Corrêia, AF, Melo, IWA, Rodrigues, RB, Boscolo, WR 2016. Digestibilidade aparente de farinha de carne e ossos de peixe para tilápia do Nilo. Archivos de Zootecnia, 65(251):34 1-348.

Gondwe, MJ, Guildford, SJ, e Hecky, RE 2011. Carregamentos de carbono, nitrogênio e fósforo de gaiolas tilápia peixes no Lago Malawi e fatores que influenciam sua magnitude. Journal of Great Lakes Research, 37, 93-101.

Hennemann, M.C. \& Petrucio, M.M 2011. Spatial and temporal dynamic of trophic relevant parameters in a subtropical coastal lagoon in Brazil. Environmental Monitoring Assessement, 181:347-361.

Koroleff, F 1976 Determination of nutrients. In: Grasshoff, K. (ed) Methods of seawater Analysis. Verlag Chemie Weinhein. p.117-181.

Kubitza, F 2000. Tilápia: Tecnologia e Planejamento na produção de Comercial. Jundiaí, 285p.

Lamparelli, MC 2004. Grau de trofia em corpos d'água do Estado de São Paulo: Avaliação de métodos de monitoramento, São Paulo 235p. (Tese de Doutorado. Instituto de Biociências da Universidade de São Paulo em: (http.// ib.usp.br/ensino/pos-graduação> Acesso em: 12 jun 2015.

Leonardo, AF, Correa, CF, Baccarin, AL 2011. Qualidade da água de um reservatório submetido à criação de tilápias em tanques-rede, no sul de São Paulo, Brasil. Boletim Instituto de Pesca, 37(4): 341-354.

Leonardo, A.F, Romagosa, E, Batlouni, S.R, Borella, M.I. 2006. Occurrence and significance of ovarian and follicular regression and follicular cachara, Pseudoplatystoma fasciatum. Arquivo Brasileiro de Medicina Veterinária e Zootecnia (Online), The Netherlands, v. 58, p. 831-840.

Koroleff, F 1976. Determination of total phosphorus. Methods of Seawater. Verlag Chemie, Weinheim, v. 168172.

Macedo, CF \& Sipauba-Tavares, LH 2010. Eutrofização e qualidade da água na piscicultura: consequências e recomendações. Bol. Inst. Pesca, 36(2), 149-163.

Malheiros, CH, Hardoim, EL, de Lima, ZM, \& Amorim, RSS 2012. Quality of water of a dam located in an agricultural area (Campo Verde, MT, Brazil). Revista Ambiente \& Água, 7(2), 245.

Marker, FH, Nusch, EA, Rai, H 1980. The measurement of photosynthetic pigments in freshwater and standartization of methods: Archive Hydrobiologie, Sttuttgart, 14, 91 - 106.

Montanhini Neto, R, \& Ostrensky, A 2015. Nutrient load estimation in the waste of Nile tilapia Oreochromis niloticus (L.) reared in cages in tropical climate conditions. Aquaculture Research, 46(6), 1309-1322.

Mudroch, A e Macknight, SD 1991. CRC Handbooks of techniques for aquatic sediments sampling. CRC Press Inc 210p.

Nusch, EA 1980. Comparison of different methods for Chlorophyll and phalopigments determination. Archiv Hydrobiologogie, 14: 14-36.

Nunes, MV, Ferreira, MJ, \& do Nascimento, RR 2013. Estudo das diferenças das variáveis abióticas de um riacho de pequena ordem e de uma represa. Periódico Eletrônico Fórum Ambiental da Alta Paulista, 9(2).

Popma, TL and Lovshin, LL 1996. World wide prospects for commercial production of tilapia. Res. Develop. Series, 41: 1-23.

Rabassó, M, \& Hernández, JM 2015. Bioeconomic analysis of the environmental impact of a marine fish farm. Journal of environmental management, 158, 24-35.

Romagosa, E, Paiva, P, Godinho, H. M. , Talmelli, EA 2003. Biologia reprodutiva de fêmeas de cachara, Pseudoplatystoma fasciatum mantidas em cativeiro. Boletim do Instituto de Pesca, São Paulo, v 29, n.2, p 151-159.

Tovar, A, Moreno, C, Mánuel-vez, MP, García-vargas, M 2000. Environmental impacts of intensive aquaculture in marine waters. Water Resource, 34(1), 334.

Wetzel, RG 2001. Limnology: lake and river ecosystems. San Diego: Academic Press. 1006. 